$\mathrm{KN}-15$

\section{Porous Crystalline Architectures: Ultra- fast Molecular Rotors and Dynamics Control by Gas Stimuli}

Angiolina Comotti ${ }^{1}$, Fabio Castiglioni ${ }^{1}$, Silvia Bracco $^{1}$, Alessandro

Pedrini ${ }^{1}$, Jacopo Perego ${ }^{1}$

1. Department of Materials Science, Milan, Italy

email: angiolina.comotti@unimib.it

A challenging issue is the dynamics of nanoporous solids after the insertion of molecular rotors in their building blocks, promising access to the control of rotary motion by chemical and physical stimuli.[1] The combination of porosity with ultra-fast rotor dynamics was discovered in molecular crystals, covalent organic frameworks and MOFs by ${ }^{2} \mathrm{H}$ spin-echo NMR spectroscopy and $\mathrm{T}_{1}$ relaxation times.[2-5] The rotors, as fast as $10^{11} \mathrm{~Hz}$ at $150 \mathrm{~K}$, are exposed to the crystalline channels, which absorb $\mathrm{CO}_{2}$ and $\mathrm{I}_{2}$ from the gas phase, even at low pressures. Interestingly, the rotor dynamics can be switched on and off by vapor absorption/desorption, showing a remarkable change of material dynamics, which, in turn, produces a modulated physical response. Novel mesoporous organosiloxane frameworks allowed us to realize periodic architectures of fast molecular rotors on which $\mathrm{C}-\mathrm{F}$ dipoles are mounted.[6] These dipolar rotors showed not only rapid dynamics $\left(10^{9} \mathrm{~Hz}\right.$ at 325 $\mathrm{K})$ in the solid-state NMR experiments, but also a dielectric response typical of a fast dipole reorientation. Moreover, crystals with permanent porosity were exploited in an unusual way to decorate crystal surfaces with regular arrays of dipolar rotors. The inserted molecules carry alkyl chains which are included as guests into the channel-ends. [7] The rotors stay at the surface due to a bulky molecular stopper which prevents the rotors from entering the channels. The host-guest relationships were established by 2D solid-state NMR and GIAO HF ab initio calculations. In a final example, flexible molecular crystals were fabricated by a series of shape-persistent azobenzene tetramers that form porous molecular crystals in their trans configuration. The efficient trans $\rightarrow$ cis photoisomerization of the azobenzene units converts the crystals into a non-porous phase but crystallinity and porosity are restored upon $\mathrm{Z} \rightarrow \mathrm{E}$ isomerization promoted by visible light irradiation or heating. We demonstrated that the photoisomerization enables reversible on/off switching of optical properties as well as the capture of $\mathrm{CO}_{2}$ from the gas phase.[8] We would like to thank Cariplo Foundation, Lombardy Region/INSTM Consortium and MIUR (PRIN 2016).

References:

[1] Comotti, A. et al. (2016). Acc. Chem. Res. 49, 1701-1710.

[2] Bracco, S. et al. (2017). Chem. Eur. J. 23, 11210.

[3] Comotti, A. et al. (2014). J. Am. Chem. Soc. 136, 618.

[4] Comotti, A. et al. (2014). Angew. Chem. Int. Ed. 53, 1043-1047.

[5] Bracco, S. et al. (2017). Chem. Comm. 53, 7776-7779.

[6] Bracco, S. et al. (2015). Angew. Chem. Int Ed. 54, 4773-4777.

[7] Kobr, L. et al. (2012). J. Am. Chem. Soc. 134, 10122-10131.

[8] Baroncini, M. et al. (2015). Nature Chem. 7, 634-640.
$\mathrm{KN}-16$

\section{Transforming co-crystals from form to function: Fact, fancy, and great expectations}

Christer Aakeroy ${ }^{1}$

1. Department of Chemistry, Kansas State University, Manhattan, KS, United States of America

email: aakeroy@ksu.edu

The precise orientation and organization of molecules in a crystalline lattice determine many bulk properties of the substance, e.g. density, solubility, thermal stability, hygroscopicity, and mechanical strength. Consequently, if we are seeking to fine-tune or 'dial-in' physical properties through a bottom-up approach, then we need to acquire control over the metrics and topologies of the structural landscape that is inhabited by a particular target molecule. Unfortunately, this presents considerable challenges because all molecular recognition events are the result of a delicate balancing act between relatively weak and reversible non-covalent interactions.

One of the reasons why co-crystals may offer unique opportunities for deliberate adjustments of bulk properties is that within a series of co-crystals of a target molecule, it may be possible to make modular changes to the crystalline framework that contains the "active" molecules. This, in turn, may allow us to make incremental changes to physical properties of a substance without having to alter the molecular properties of the target. The inexorable (but not always obvious) connections between structure and properties means that with the ability to change specific aspects of the solid form of a compound comes new and unique prospects for materials design.

This presentation will describe different strategies for the design and practical synthesis of co-crystals using both hydrogen- and halogen bonds, and will also offer several examples of co-crystal technology as applied to both energetic materials and agrochemicals.

References:

[1] Sandhu, B., Sinha, A.S., Desper, J., \& Aakeröy, C.B., (2018),

Chem Commun., in press.

[2] Aakeröy, C.B., (2015). Acta Cryst, B71, 387-391.

[3] Aakeröy, C.B.; Wijethunga, T.K. \& Desper, J., (2015) Chem. Eur. J. 21, $11029-11037$.

Keywords: Co-crystals, intermolecular interactions, materials 